\title{
Nonlinear MDOF system Survival Probability Determination Subject to Evolutionary Stochastic Excitation
}

\author{
Ioannis P. Mitseas $14^{*}$ - Ioannis A. Kougioumtzoglou ${ }^{2}$ - Pol D. Spanos 3 - Michael Beer1,4,5 \\ ${ }^{1}$ Leibniz University Hannover, Faculty of Civil Engineering and Geodetic Science, Germany \\ 2 Columbia University, Department of Civil Engineering and Engineering Mechanics, USA \\ ${ }^{3}$ Rice University, Department of Mechanical Engineering, USA \\ 4 University of Liverpool, Institute for Risk and Uncertainty and School of Engineering, United Kingdom \\ 5 Tongji University, School of Civil Engineering and Shanghai Institute of Disaster Prevention and Relief, China
}

\begin{abstract}
An approximate technique for assessing the reliability of nonlinear multi-degree-of-freedom (MDOF) systems subject to a non-stationary stochastic excitation vector is developed. The proposed technique can be construed as a two-stage approach. First, relying on statistical linearization and utilizing a dimension reduction approach the nonlinear $n$-degree-of-freedom system is decoupled and cast into (n) effective single-degree-of-freedom (SDOF) linear time-variant (LTV) oscillators. Second, utilizing the effective SDOF LTV oscillator time-varying stiffness and damping elements in conjunction with a stochastic averaging treatment of the problem, the MDOF system survival probability and firstpassage PDF are determined. Overall, the developed technique appears to be efficient and versatile since it can handle readily, at a low computational cost, a wide range of nonlinear/hysteretic behaviors as well as various stochastic excitation forms, even of the fully nonstationary in time and frequency kind. A 3-DOF system exhibiting hysteresis following the Bouc-Wen model is included in the numerical examples section. Comparisons with pertinent Monte Carlo simulations demonstrate the accuracy of the technique.
\end{abstract}

Keywords: first-passage problem, nonlinear stochastic dynamics, evolutionary stochastic processes, nonlinear/hysteretic systems, survival probability

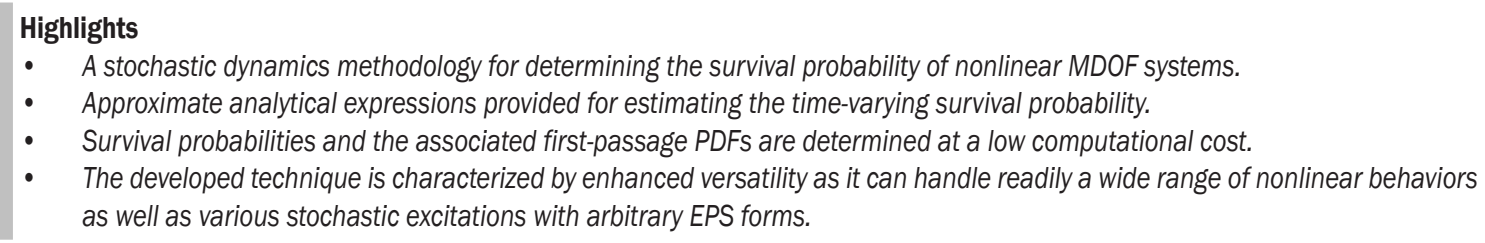

\section{INTRODUCTION}

Excitations acting upon dynamical systems such as wind, wave, and seismic loads commonly exhibit evolutionary features. In this setting, not only the intensity of the excitation but also its frequency content exhibit strong variability. This fact necessitates the representation of this class of structural loads by non-stationary stochastic processes. Further, structural systems under severe excitations can exhibit significant nonlinear behavior of the hysteretic kind. Thus, of particular interest to the structural dynamics community is the development of techniques for determining the response and assessing the reliability of nonlinear/hysteretic systems subject to evolutionary stochastic excitations (e.g., [1] to [3]).

Further, in engineering dynamics, the evaluation of the probability that the system response stays within prescribed limits for a specified time interval is advantageous for reliability based system design applications. In this regard, the first-passage problem, that is, the determination of the above time-variant probability known as survival probability, has been a persistent challenge in the field of stochastic dynamics for many decades.

Monte Carlo simulation techniques are among the most potent tools for assessing the reliability of a system (e.g. [4]). Nevertheless, there are cases where the computational cost of these techniques can be prohibitive, especially when large-scale complex systems are considered; thus, rendering the development of alternative efficient approximate analytical/numerical techniques for addressing the first-passage problem necessary. Indicatively, one of the early approaches, restricted to linear systems, relies on the knowledge of the mean up-crossing rates and on Poisson distribution based approximations (e.g., [5] to [7]). Further attempts to address the firstpassage problem range from analytical ones (e.g., [8]) to numerical ones (e.g., [9]). Furthermore, techniques based on the concepts of the numerical path integral (e.g., [10] to [13]), of the probability density evolution (e.g., [3]), or of stochastic averaging/linearization (e.g., [14]) constitute some of the more recent approaches. 
In this paper, an approximate analytical technique for determining the survival probability and firstpassage probability density function (PDF) of nonlinear multi-degree-of-freedom (MDOF) systems subject to an evolutionary stochastic excitation vector is developed. Specifically, first relying on a statistical linearization based dimension reduction approach the original MDOF system is decoupled and cast into $(n)$ effective single-degree-of-freedom (SDOF) linear time-variant (LTV) systems corresponding to each and every degree of freedom of the original MDOF system. Second, a stochastic averaging based approximate technique is utilized to derive the nonlinear MDOF system survival probability and first-passage PDF at a low computational cost.

The remainder of this paper is organized as follows: In section 1.1 the statistical linearization technique for nonlinear MDOF systems is presented. Next, in section 1.2 a stochastic averaging/statistical linearization treatment of the problem, through a system dimension reduction approach is briefly delineated. In section 1.3, it is shown that the nonlinear MDOF system non-stationary marginal, transition and the joint response amplitude probability density functions (PDFs) can be approximated by closed-form expressions. Further, section 2 provides analytical closed-form expressions for the time-dependent survival probability of the nonlinear MDOF structural system as well as for the corresponding first-passage PDF. In section 3, illustrative examples comprising a 3-DOF system exhibiting Bouc-Wen hysteresis and subject to evolutionary stochastic excitations are considered. Pertinent MCS data demonstrate the reliability of the proposed technique. Finally, section 4 provides with concluding remarks.

\section{MDOF SYSTEM DIMENSION REDUCTION}

In this section, the basic elements of an approximate dimension reduction/decoupling technique developed by some of the authors for determining the nonstationary response amplitude PDF of nonlinear MDOF systems subject to evolutionary stochastic excitation are reviewed for completeness; see [15] and [16] for a more detailed presentation.

\subsection{Statistical Linearization Treatment}

Consider an $n$-degree-of-freedom nonlinear system governed by the equation:

$$
M \ddot{y}+C \dot{y}+K \boldsymbol{y}+\boldsymbol{g}(\boldsymbol{y}, \dot{\boldsymbol{y}})=\boldsymbol{F}(t) .
$$

where $\ddot{\boldsymbol{y}}$ denotes the response acceleration vector, $\dot{\boldsymbol{y}}$ is the response velocity vector, $\boldsymbol{y}$ is the response displacement vector, defined in relative coordinates; $\boldsymbol{M}, \boldsymbol{C}$ and $\boldsymbol{K}$ denote the $(n \times n)$ mass, damping and stiffness matrices, respectively; $\boldsymbol{g}(\boldsymbol{y}, \dot{\boldsymbol{y}})$ is an arbitrary nonlinear $(n \times 1)$ vector function of the variables $\boldsymbol{y}$ and $\dot{\boldsymbol{y}} \cdot \boldsymbol{F}(t)^{T}=\left(f_{1}(t), f_{2}(t), \ldots, f_{n}(t)\right)$ is a $(n \times 1)$ zero mean, non-stationary stochastic excitation vector process defined as $\boldsymbol{F}(t)=\boldsymbol{\gamma} \ddot{a}(t)$ where $\boldsymbol{\gamma}^{T}=\left(\gamma_{1}, \gamma_{2}, \ldots, \gamma_{n}\right)$ is an arbitrary $(n \times 1)$ vector of constant weighting coefficients, and $\ddot{a}(t)$ is a nonstationary process with an evolutionary power spectrum (EPS) $S_{\ddot{a}}(\omega, t)$. In this regard, $\boldsymbol{F}(t)$ possesses the EPS matrix:

$$
\boldsymbol{S}_{\boldsymbol{F}}(\boldsymbol{\omega}, \boldsymbol{t})=\left[\begin{array}{cccc}
\gamma_{1}^{2} S_{\ddot{a}}(\omega, t) & 0 & \cdots & 0 \\
0 & \gamma_{2}^{2} S_{\ddot{a}}(\omega, t) & \cdots & 0 \\
\vdots & \vdots & \ddots & \vdots \\
0 & 0 & \cdots & \gamma_{n}^{2} S_{\ddot{a}}(\omega, t)
\end{array}\right] .(2)
$$

Further, the non-stationary stochastic excitation process is regarded to be a filtered stationary stochastic process according to the concept proposed by Priestley [17]; see also [18]. Thus, the excitation EPS matrix of Eq. (2) takes the form:

$$
\boldsymbol{S}_{F}(\omega, t)=\boldsymbol{A}(\omega, t) \boldsymbol{S}_{\tilde{F}}(\omega) \boldsymbol{A}(\omega, t)^{T^{*}},
$$

where the superscripts $(T)$ and $(*)$ denote matrix transposition and complex conjugation, respectively; $\boldsymbol{A}(\omega, t)$ is the modulating matrix which serves as a time-variant filter; and $\boldsymbol{S}_{\tilde{F}}(\omega)$ is the power spectrum matrix corresponding to the stationary stochastic vector process $\tilde{\boldsymbol{F}}(t)$. Note that both separable and non-separable EPS can be defined considering Eq. (3). In this manner, excitations exhibiting variability in both the intensity and the frequency content can be considered. Focusing next on the frequency domain, the response determination problem is defined as seeking the corresponding system response EPS matrix of the form:

$$
\begin{aligned}
& S_{y}(\omega, t)= \\
& =\left[\begin{array}{cccc}
S_{y_{1} y_{1}}(\omega, t) & S_{y_{1} y_{2}}(\omega, t) & \ldots & S_{y_{1} y_{n}}(\omega, t) \\
S_{y_{2} y_{1}}(\omega, t) & S_{y_{2} y_{2}}(\omega, t) & \ldots & \vdots \\
\vdots & \vdots & \ddots & S_{y_{n-1} y_{n}}(\omega, t) \\
S_{y_{n} y_{1}}(\omega, t) & \ldots & S_{y_{n} y_{n-1}}(\omega, t) & S_{y_{n} y_{n}}(\omega, t)
\end{array}\right] .(4)
\end{aligned}
$$

According to the statistical linearization method (e.g., [1] to [3]), a linearized version of Eq. (1) takes the form: 


$$
\boldsymbol{M} \ddot{\boldsymbol{y}}+\left(\boldsymbol{C}+\boldsymbol{C}_{e q}\right) \dot{\boldsymbol{y}}+\left(\boldsymbol{K}+\boldsymbol{K}_{e q}\right) \boldsymbol{y}=\boldsymbol{F}(t) .
$$

Next, adopting the standard assumption that the response processes are Gaussian, the time-dependent elements of the equivalent linear matrices $\boldsymbol{C}_{\boldsymbol{e q}}$ and $\boldsymbol{K}_{\boldsymbol{e q}}$ are given by the expressions:

and

$$
c_{i, j}^{e q}=E\left\{\frac{\partial g_{i}}{\partial \dot{y}_{j}}\right\},
$$

$$
k_{i, j}^{e q}=E\left\{\frac{\partial g_{i}}{\partial y_{j}}\right\} \text {. }
$$

Further, for a linear MDOF system subject to evolutionary stochastic excitation a matrix inputoutput spectral relationship of the form:

$$
\boldsymbol{S}_{y}(\omega, t)=\boldsymbol{H}_{g e n}(\omega, t) \boldsymbol{S}_{\tilde{F}}(\omega) \boldsymbol{H}_{g e n}^{T^{*}}(\omega, t)
$$

can be derived (e.g., [1] and [3]), where

$$
\boldsymbol{H}_{g e n}(\omega, t)=\int_{0}^{t} \boldsymbol{h}(t-\tau) \boldsymbol{A}(\omega, \tau) e^{-i \omega(t-\tau)} d \tau .
$$

In Eq. (9) denotes the impulse response function matrix. Furthermore, the time dependent crossvariance of the response can be evaluated by the expression:

$$
E\left[y_{i} y_{j}\right]=\int_{-\infty}^{\infty} S_{y_{i} y_{j}}(\omega, t) d \omega
$$

It can be readily seen that Eqs. (6) to (10) constitute a coupled nonlinear system of algebraic equations to be solved numerically for the system response covariance matrix. Note in passing that instead of the frequency domain Wiener-Khinchin relationship of Eq. (8), a state-variable formulation can be adopted yielding a system of differential equations of the Lyapunov kind (e.g., [1] and [19]) for the system response covariance matrix. Nevertheless, although a pre-filtering treatment can be applied for considering non-stationary stochastic excitation processes of the separable kind (e.g., [1]), excitations possessing a non-separable EPS (e.g. realistic cases of earthquake excitations) cannot be accounted for, at least in a straightforward manner. Next, omitting the convolution of the impulse response function matrix with the modulating matrix can lead to substantial reduction of computational effort, especially for the case of MDOF systems (e.g., [16] and [20]). In this manner, Eq. (9) takes the form:

$$
\boldsymbol{H}_{\text {gen }}(\omega, t)=\boldsymbol{H}(\omega) \boldsymbol{A}(\omega, t),
$$

where $\boldsymbol{H}(\omega)$ is the frequency response function (FRF) matrix defined as:

$$
\boldsymbol{H}(\omega)=\left(-\omega^{2} \boldsymbol{M}+i \omega\left(\boldsymbol{C}+\boldsymbol{C}_{e q}\right)+\left(\boldsymbol{K}+\boldsymbol{K}_{e q}\right)\right)^{-1}
$$

Consequently, taking into account Eqs. (3) and (8), Eq. (11) becomes:

$$
\boldsymbol{S}_{y}(\omega, t)=\boldsymbol{H}(\omega) \boldsymbol{S}_{F}(\omega, t) \boldsymbol{H}^{T^{*}}(\omega)
$$

Note that the Eq. (13) can be regarded as a quasistationary approximate relationship which, in general, yields satisfactory accuracy in cases of relatively stiff systems (e.g., [20] to [22]). Note in passing that the spectral input-output relationship of Eq. (13) is exact for the case of stationary processes (e.g., [1] to [3]). Further, adopting the aforementioned quasi-stationary approach, it can be readily seen that for the $i^{\text {th }}$ degree of freedom, using Eqs. (2), (10) and (13) yields:

$$
\begin{aligned}
E\left[y_{i}^{2}(t)\right] & =\int_{-\infty}^{\infty}\left(\left|H_{i 1}(\omega)\right|^{2} \gamma_{1}^{2}+\ldots\right. \\
& \left.+\left|H_{i n}(\omega)\right|^{2} \gamma_{n}^{2}\right) S_{\ddot{a}}(\omega, t) d \omega,
\end{aligned}
$$

and

$$
\begin{aligned}
E\left[\dot{y}_{i}^{2}(t)\right] & =\int_{-\infty}^{\infty} \omega^{2}\left(\left|H_{i 1}(\omega)\right|^{2} \gamma_{1}^{2}+\ldots\right. \\
& \left.+\left|H_{i n}(\omega)\right|^{2} \gamma_{n}^{2}\right) S_{\ddot{a}}(\omega, t) d \omega,
\end{aligned}
$$

Eqs. (14) and (15) hold true in the approximate quasi-stationary sense delineated earlier. Clearly, Eq. (13) constitutes an approximate formula for determining the MDOF system response EPS matrix at a low computational cost; thus, circumventing computationally intensive Monte Carlo simulations.

\subsection{Effective SDOF Linear Time-Variant System}

Following next the system dimension reduction approach developed in [16], an auxiliary effective SDOF LTV system corresponding to the $i^{\text {th }}$ degree of freedom can be defined as:

$$
\ddot{y}_{i}+\beta_{e q, i}(t) \dot{y}_{i}+\omega_{e q, i}^{2}(t) y_{i}=\ddot{\alpha}_{i}(t),
$$

where the time-varying equivalent stiffness and damping elements of the effective LTV system can be determined by equating the variances of the response displacement and velocity expressed utilizing the quasi-stationary FRF of Eq. (16) with the 
corresponding ones determined via Eqs. (14) and (15); this yields:

$$
\begin{aligned}
& E\left[y_{i}^{2}(t)\right]= \\
& =\int_{-\infty}^{\infty}\left(\frac{1}{\left(\omega_{e q, i}^{2}(t)-\omega^{2}\right)^{2}+\left(\beta_{e q, i}(t) \omega\right)^{2}}\right) \times \gamma_{i}^{2} S_{a ̈}(\omega, t) d \omega,(17)
\end{aligned}
$$

and

$$
\begin{aligned}
& E\left[\dot{y}_{i}^{2}(t)\right]= \\
& =\int_{-\infty}^{\infty} \omega^{2}\left(\frac{1}{\left(\omega_{e q, i}^{2}(t)-\omega^{2}\right)^{2}+\left(\beta_{e q, i}(t) \omega\right)^{2}}\right) \times \\
& \times \gamma_{i}^{2} S_{\ddot{a}}(\omega, t) d \omega,
\end{aligned}
$$

Clearly, Eqs. (17) and (18) in conjunction with Eqs. (14) and (15) constitute a nonlinear system of two algebraic equations to be solved for the evaluation of the LTV system time-varying equivalent stiffness $\omega_{e q, i}^{2}(t)$ and damping $\beta_{e q, i}(t)$ coefficients. Note that determining the time-varying natural frequency $\omega_{e q, i}(t)$ is especially important for a number of reasons such as tracking and avoiding moving resonance phenomena (e.g., [23]), determining peak system response estimates based on design spectrum compatible excitation power spectra (e.g., [24]), or developing efficient approximate techniques for determining nonlinear system survival probability and first-passage PDF (e.g., [14]).

Next, a stochastic averaging technique (e.g., [15] and [16]) is applied for casting the second-order stochastic differential equation (SDE) of Eq. (1) into a first-order SDE governing the evolution in time of the response amplitude $a_{i}(t)$. In this regard, and based primarily on the assumption of light damping, it can be argued that the response $y_{i}(t)$ of the effective LTV system of Eq. (16) exhibits a pseudo-harmonic behavior described by the equations:

$$
y_{i}(t)=a_{i}(t) \cos \left(\omega_{e q, i}(t) t+\varphi_{i}(t)\right),
$$

and

$$
\dot{y}_{i}(t)=-\omega_{e q, i}(t) a_{i}(t) \times \sin \left(\omega_{e q, i}(t) t+\varphi_{i}(t)\right) .
$$

In Eq. (19) the response amplitude $a_{i}(t)$ is a slowly varying function with respect to time defined as:

$$
a_{i}^{2}(t)=y_{i}^{2}(t)+\left(\frac{\dot{y}_{i}(t)}{\omega_{e q, i}(t)}\right)^{2},
$$

whereas $\varphi_{i}(t)$ stands for the phase of the response $y_{i}(t)$. Further, relying on a combination of deterministic and stochastic averaging (e.g., [16]) a first-order SDE governing each and every degree-of-freedom response amplitude process $a_{i}(t)$ takes the form:

$$
\begin{aligned}
\dot{a}_{i}(t)= & -\frac{1}{2} \beta_{e q, i}(t) \alpha_{i}(t)+\frac{\pi S_{F}\left(\omega_{e q, i}(t), t\right)}{2 \alpha_{i}(t) \omega_{e q, i}^{2}(t)}+ \\
& +\frac{\sqrt{\pi S_{F}\left(\omega_{e q, i}(t), t\right)}}{\omega_{e q, i}(t)} \eta(t) .
\end{aligned}
$$

In Eq. (22), $\eta(t)$ stands for a stationary, zero mean and delta correlated Gaussian white noise process of unit intensity, i.e., $E(\eta(t))=0$; and $E(\eta(t) \eta(t+\tau))=\delta(\tau)$, with $\delta(\tau)$ being the Dirac delta function. Associated with the above SDE (Eq. (22)) is the Fokker-Planck (F-P) partial differential equation governing the response amplitude transition PDF of the Markovian process $\alpha_{i}$; that is,

$$
\begin{aligned}
& \frac{\partial}{\partial t} p\left(a_{i, 2}, t_{2} \mid a_{i, 1}, t_{1}\right)= \\
& =-\frac{\partial}{\partial \alpha_{i}}\left[\left(-\frac{1}{2} \beta_{e q, i}(t) \alpha_{i}+\frac{\pi S_{F}\left(\omega_{e q, i}(t), t\right)}{2 \alpha_{i} \omega_{e q, i}^{2}(t)}\right) p\left(a_{i, 2}, t_{2} \mid a_{i, 1}, t_{1}\right)\right]+ \\
& +\frac{1}{2} \frac{\partial^{2}}{\partial \alpha_{i}^{2}}\left[\left(\frac{\pi S_{F}\left(\omega_{e q, i}(t), t\right)}{\omega_{e q, i}^{2}(t)}\right) p\left(a_{i, 2}, t_{2} \mid a_{i, 1}, t_{1}\right)\right]
\end{aligned}
$$

Further, considering the case $p\left(a_{i, 2}, t_{2} \mid a_{i, 1}=0\right.$, $\left.t_{1}=0\right)=p\left(a_{i}, t\right)$, the marginal system response amplitude PDF has been shown to follow a timedependent Rayleigh distribution of the form (e.g., [16], [25] and [26]):

$$
p\left(a_{i}, t\right)=\frac{a_{i}}{c_{i}(t)} \exp \left(-\frac{a_{i}^{2}}{2 c_{i}(t)}\right),
$$

where $c_{i}(t)$ accounts for the non-stationary variance of the LTV system of Eq. (16). As it was shown in [15] and [16] substituting Eq. (24) into Eq. (23) and manipulating yields the following nonlinear ordinary differential equation (ODE):

$$
\dot{c}_{i}(t)=-\beta_{e q, i}(t) c_{i}(t)+\frac{\pi S_{F}\left(\omega_{e q, i}(t), t\right)}{\omega_{e q, i}^{2}(t)},
$$

to be solved for the non-stationary LTV system response variance $c_{i}(t)$ via standard numerical schemes such as the fourth order Runge-Kutta.

\subsection{Transition and Joint Nonlinear System Response PDFs}

Taking into account that no change of state can occur if the transition time is zero i.e., $p\left(a_{i, 2}, t_{1} \mid a_{i, 1}, t_{1}\right)=\delta\left(a_{i, 2}-a_{i, 1}\right)$ and following a similar analysis as the one in [25], the transition response amplitude PDF $p\left(a_{i, 2}, t_{2} \mid a_{i, 1}, t_{1}\right)$ for the $i^{\text {th }}$ degree-of- 
freedom of the original MDOF system is assumed to be of the form:

$$
\begin{aligned}
& p\left(a_{i, 2}, t_{2} \mid a_{i, 1}, t_{1}\right)= \\
& \frac{a_{i, 2}}{c_{i}\left(t_{1}, t_{2}\right)} \exp \left(-\frac{a_{i, 2}^{2}+h_{i}^{2}\left(t_{1}, t_{2}\right)}{2 c_{i}\left(t_{1}, t_{2}\right)}\right) I_{0} \times\left(\frac{a_{i, 2} h_{i}\left(t_{1}, t_{2}\right)}{c_{i}\left(t_{1}, t_{2}\right)}\right),
\end{aligned}
$$

where $c_{i}\left(t_{1}, t_{2}\right)$ and $h_{i}\left(t_{1}, t_{2}\right)$ are functions to be determined and $I_{0}$ represents the modified Bessel function of the first kind and of zero order. Next, substituting Eq. (26) into the F-P Eq. (23) and manipulating (see also [9],[14] and [25]) yields the linear first-order ODEs:

$$
\begin{aligned}
& \frac{d c_{i}\left(t_{1}, t_{2}\right)}{d t_{2}}+\beta_{e q, i}\left(c_{i}\left(t_{1}, t_{2}\right)\right) c_{i}\left(t_{1}, t_{2}\right)- \\
& -\frac{\pi S_{F}\left(\omega_{e q, i}\left(c_{i}\left(t_{1}, t_{2}\right)\right), t_{2}\right)}{\omega_{e q, i}^{2}\left(c_{i}\left(t_{1}, t_{2}\right)\right)}=0
\end{aligned}
$$

and

$$
\frac{d h_{i}\left(t_{1}, t_{2}\right)}{d t_{2}}+\frac{1}{2} \beta_{e q, i}\left(h_{i}\left(t_{1}, t_{2}\right)\right) h_{i}\left(t_{1}, t_{2}\right)=0
$$

Relying on the assumption that the equivalent damping and stiffness coefficients follow a slowly varying with respect to time behavior, the following approximations over a small time interval $\left[t_{i, j-1}, t_{i, j}\right]$ are introduced; i.e., $\quad \beta_{\text {eq, } i}\left(t_{i, j}\right)=\beta_{\text {eq, } i}\left(t_{i, j-1}\right)$ and $\omega_{\text {eq, } i}\left(t_{i, j}\right)=\omega_{\text {eq,i } i}\left(t_{i, j-1}\right)$ for $t \in\left[t_{i, j-1}, t_{i, j}\right]$. Next, based on the slowly varying with time behavior of the EPS, $S_{f_{i, i}}(\omega, t)$ is also treated as a constant over the interval $\left[t_{i, j-1}, t_{i, j}\right]$. Further, based on the above assumptions, introducing the variable $\tau_{i, j}=t_{i, j}-t_{i, j-1}$, and applying a first-order Taylor expansion around the point $\tau_{i, j}=0$, Eqs. (27) and (28) become (see [14] for a detailed derivation):

$c_{i}\left(t_{i, j-1}, t_{i, j}\right)=\pi S_{F}\left(\omega_{e q, i}\left(t_{i, j-1}\right), t_{i, j-1}\right) \frac{\tau_{i, j}}{\omega_{e q, i}^{2}\left(t_{i, j-1}\right)}$,

and

$$
h_{i}\left(t_{i, j-1}, t_{i, j}\right)=a_{i, j-1} \sqrt{1-\beta_{e q, i}\left(t_{i, j-1}\right) \tau_{i, j}} .
$$

Furthermore, considering Eqs. (25) and (29) and applying a first-order Taylor expansion for the response variance $c_{i}(t)$ around the point $t=t_{i, j-1}$ yields:

$$
c_{i}\left(t_{i, j-1}, t_{i, j}\right)=c_{i}\left(t_{i, j}\right)-c_{i}\left(t_{i, j-1}\right)\left(1-\beta_{e q, i}\left(t_{i, j-1}\right) \tau_{i, j}\right)
$$

Relying next on the Markovian assumption for the process $a_{i}$, the joint-response amplitude PDF $p\left(a_{i, j-1}, t_{j-1} ; a_{i, j}, t_{j}\right)$ is given by:

$p\left(a_{i, j-1}, t_{j-1} ; a_{i, j}, t_{j}\right)=p\left(a_{i, j-1}, t_{j-1}\right) p\left(a_{i, j}, t_{j} \mid a_{i, j-1}, t_{j-1}\right)$.

Utilizing Eqs. (24) and (26), Eq. (32) becomes:

$$
\begin{aligned}
& p\left(\alpha_{i, j-1}, t_{j-1} ; \alpha_{i, j}, t_{j}\right)=\frac{\alpha_{i, j-1} \alpha_{i, j}}{c_{i}\left(t_{j-1}\right) c_{i}\left(t_{j-1}, t_{j}\right)} \times \\
& \exp \left(\frac{-\alpha_{i, j}^{2} c_{i}\left(t_{j-1}\right)-\alpha_{i, j-1}^{2} c_{i}\left(t_{j-1}, t_{j}\right)}{2 c_{i}\left(t_{j-1}\right) c_{i}\left(t_{j-1}, t_{j}\right)}-\right. \\
& \left.\frac{h_{i}^{2}\left(t_{j-1}, t_{j}\right) c_{i}\left(t_{j-1}\right)}{2 c_{i}\left(t_{j-1}\right) c_{i}\left(t_{j-1}, t_{j}\right)}\right) I_{0}\left(\frac{\alpha_{i, j} h_{i}\left(t_{j-1}, t_{j}\right)}{c_{i}\left(t_{j-1}, t_{j}\right)}\right) .
\end{aligned}
$$

Further, setting

$$
r_{i, j}^{2}=\frac{c_{i}\left(t_{i, j-1}\right)}{c_{i}\left(t_{i, j}\right)}\left(1-\beta_{e q, i}\left(t_{i, j-1}\right) \tau_{i, j}\right),
$$

Eq. (31) yields:

$$
c_{i}\left(t_{i, j-1}, t_{i, j}\right)=c_{i}\left(t_{i, j}\right)\left(1-r_{i, j}^{2}\right) .
$$

Next, considering Eqs. (29) and (30) and Eqs. (34) and (35), the joint response amplitude PDF $p\left(a_{i, j-1}, t_{i, j-1} ; a_{i, j}, t_{i, j}\right)$ of Eq. (33) is given in the form:

$$
\begin{aligned}
& p\left(\alpha_{i, j-1}, t_{i, j-1} ; \alpha_{i, j}, t_{i, j}\right)=\frac{\alpha_{i, j-1} \alpha_{i, j}}{c_{i}\left(t_{i, j-1}\right) c_{i}\left(t_{i, j}\right)\left(1-r_{i, j}^{2}\right)} \times \\
& \times \exp \left(-\frac{\alpha_{i, j}^{2} c_{i}\left(t_{i, j-1}\right)+\alpha_{i, j-1}^{2} c_{i}\left(t_{i, j}\right)}{2 c_{i}\left(t_{i, j-1}\right) c_{i}\left(t_{i, j}\right)\left(1-r_{i, j}^{2}\right)}\right) I_{0} \times \\
& \times\left(\frac{\alpha_{i, j} \alpha_{i, j-1} r_{i, j}}{\left.\sqrt{c_{i}\left(t_{i, j-1}\right) c_{i}\left(t_{i, j}\right)}\right)\left(1-r_{i, j}^{2}\right)}\right) .
\end{aligned}
$$

\section{NONLINEAR MDOF SYSTEM RELIABILITY ASSESSMENT}

In this section the approximate analytical technique developed by some of the authors in [14] for nonlinear SDOF survival probability determination is generalized herein to account for MDOF systems by utilizing the dimension reduction/decoupling technique outlined in section 1 .

In this regard, the survival probability $P_{i}^{B}$ is defined as the probability that the system response 
amplitude $a_{i}$ stays below a prescribed barrier $B$ over the time interval $[0, T]$, given that $a_{i}(t=0)<B$. Further, the first-passage PDF and the survival probability $p_{i}^{B}(T)$ are related according to the expression:

$$
p_{i}^{B}(T)=-\frac{d P_{i}^{B}(T)}{d T} .
$$

Next, adopting the discretization scheme employed in [9] yields intervals of the form:

$$
\begin{gathered}
{\left[t_{i, j-1}, t_{i, j}\right], \quad j=1,2, \ldots, m, \quad t_{i, 0}=0,} \\
t_{i, m}=T, \quad t_{i, j}-t_{i, j-1}=\frac{T_{e q, i}\left(t_{i, j-1}\right)}{2},
\end{gathered}
$$

where the response amplitude $a_{i}$ is assumed to be constant over $\left[t_{i, j-1}, t_{i, j}\right]$ due to its slowly varying in time behavior. In Eq. (38) $T_{e q, i}$ represents the LTV system equivalent natural period given by:

$$
T_{e q, i}(t)=\frac{2 \pi}{\omega_{e q, i}(t)} .
$$

Note in passing that a smaller time interval can be chosen if higher accuracy is required. In this regard, the survival probability $P_{i}^{B}$ is assumed to have a constant value over the same time interval as well. Obviously, the survival probability is given by

$$
P_{i}^{B}(T)=\prod_{j=1}^{m}\left[1-F_{i, j}^{B}\right],
$$

where $F_{i, j}^{B}$ is defined as the probability that the response amplitude $a_{i}$ will exceed the prescribed barrier $B$ over the time interval $\left[t_{i, j-1}, t_{i, j}\right]$, given that no crossings have occurred prior to time $t_{i, j-1}$. Next, invoking the Markovian property of the response amplitude $a_{i}$, one gets:

$$
F_{i, j}^{B}=\frac{\operatorname{Prob}\left[a_{i}\left(t_{i, j}\right) \geq B \cap a_{i}\left(t_{i, j-1}\right)<B\right]}{\operatorname{Prob}\left[a_{i}\left(t_{i, j-1}\right)<B\right]}=\frac{H_{i, j-1, j}^{B}}{H_{i, j-1}^{B}},
$$

where $\cap$ denotes the intersection symbol. Utilizing Eq. (24) $H_{i, j-1}^{B}$ can be determined analytically in a straightforward manner; that is,

$$
H_{i, j-1}^{B}=\int_{0}^{B} p\left(a_{i, j-1}, t_{i, j-1}\right) d a_{i, j-1}=1-\exp \left(-\frac{B^{2}}{2 c_{i}\left(t_{i, j-1}\right)}\right),
$$

whereas $H_{i, j-1, j}^{B}$ is defined as a double integral of the form:

$$
H_{i, j-1, j}^{B}=\int_{B}^{\infty} d a_{i, j} \times \int_{0}^{B} p\left(a_{i, j-1}, t_{i, j-1} ; a_{i, j}, t_{i, j}\right) d a_{i, j-1} .
$$

Further, taking into account Eq. (36) and expanding the Bessel function $I_{0}(x)$ in the form (e.g., [27]):

$$
I_{0}(x)=\sum_{k=0}^{\infty} \frac{(x / 2)^{2 k}}{k ! \Gamma(\kappa+1)},
$$

analytical treatment of the involved integrals is possible yielding:

$$
H_{i, j-1, j}^{B}=A_{i, 0}+\sum_{n=1}^{N} A_{i, n},
$$

where

$$
\begin{aligned}
A_{i, 0} & =\exp \left(\frac{-B^{2}}{2 c_{i}\left(t_{i, j}\right)\left(1-r_{i, j}^{2}\right)}\right) \times \\
& \times\left(1-\exp \left(\frac{-B^{2}}{2 c_{i}\left(t_{i, j-1}\right)\left(1-r_{i, j}^{2}\right)}\right)\right) \times\left(1-r_{i, j}^{2}\right),
\end{aligned}
$$

and

$$
A_{i, n}=\frac{r_{i, j}^{2 n}}{\left(c_{i}\left(t_{i, j-1}\right) c_{i}\left(t_{i, j}\right)\right)^{n+1}\left(1-r_{i, j}^{2}\right)^{2 n+1}} \times \frac{L_{i, n}}{\prod_{n=1}^{N}(2 n)^{2}},
$$

with

$$
\begin{aligned}
& L_{i, n}=4^{n} c_{i}\left(t_{i, j-1}\right)^{n+1} c_{i}\left(t_{i, j}\right)\left(1-r_{i, j}^{2}\right)^{n+2} \times \\
& \times\left(\Gamma_{i}\left[1+n, \frac{B^{2}}{2 c_{i}\left(t_{i, j-1}\right)\left(1-r_{i, j}^{2}\right)}\right]-\Gamma_{i}[1+n, 0]\right) \times \\
& \times\left(\begin{array}{l}
-\left(c_{i}\left(t_{i, j}\right)\left(1-r_{i, j}^{2}\right)\right)^{n} \Gamma_{i}[1+n]+ \\
+\left(\frac{B^{2}}{c_{i}\left(t_{i, j}\right)\left(1-r_{i, j}^{2}\right)}\right)^{-n} \times \\
\times\left(\Gamma_{i}[1+n]-\Gamma_{i}\left[1+n, \frac{B^{2}}{2 c_{i}\left(t_{i, j}\right)\left(1-r_{i, j}^{2}\right)}\right]\right) \times B^{2 n}
\end{array}\right),
\end{aligned}
$$

In Eq. (48) $\Gamma_{i}[\gamma, z]$ represents the incomplete Gamma function defined as $\Gamma_{i}[\gamma, z]=\int_{z}^{\infty} t^{\gamma-1} e^{-t} d t$.

Concisely, the developed technique comprises the following steps:

i. Determination of the MDOF system nonstationary response covariance matrix (Eqs. (10) and (13)) via a statistical linearization treatment of the problem.

ii. Determination of the equivalent linear timevarying elements $\beta_{\text {eq,i }}(t)$ and $\omega_{\text {eq,i }}(t)$ by solving the system of algebraic equations (Eqs. (17) and (18)). 
iii. Determination of $c_{i}(t)$ via numerically integrating the first-order ODE Eq. (25).

iv. Determination of the equivalent natural period $T_{\text {eq,i }}(t)$ (Eq. (39)) and discretization of the time domain via Eq. (38).

v. Determination of the parameters $H_{i, j-1}^{B}$ and $H_{i, j-1, j}^{B}$ via Eqs. (42) and (43).

vi. Determination of the survival probability $P_{i}^{B}(T)$ via Eq. (40) and of the corresponding firstpassage PDF $p_{i}^{B}(T)$ via Eq. (37).

\section{NUMERICAL APPLICATIONS}

In this section, a nonlinear three-degree-of-freedom system following the Bouc-Wen hysteretic model (e.g., [28] and [29]) subject to evolutionary stochastic excitation is considered to demonstrate the reliability of the technique.

The survival probabilities and the first-passage PDFs obtained via the developed approximate technique are compared with survival probability and first-passage PDF estimates obtained via pertinent Monte Carlo simulations (10,000 realizations). The Monte Carlo simulations were conducted by utilizing a spectral representation methodology; additional details can be found in [30].

Further, a standard fourth-order Runge-Kutta numerical integration scheme is employed for solving the nonlinear system differential equation of motion (Eq. (1)), whereas the barrier level $B$ is expressed as a fraction $\lambda$ of the maximum over time and over DOF value of the non-stationary response displacement standard deviation, i.e. $B=\lambda \max _{i \text { and } t}\left(\sigma_{i}(t)\right)$ with $\sigma_{i}(t)=\sqrt{c_{i}(t)}$. Considering displacements defined in relative coordinates, the 3 -DOF nonlinear system is governed by Eq. (1) where

$$
\begin{aligned}
& \boldsymbol{y}^{T}=\left(\begin{array}{llllll}
y_{1} & y_{2} & y_{3} & z_{1} & z_{2} & z_{3}
\end{array}\right), \\
& M=\left[\begin{array}{ll}
M_{11} & M_{12} \\
M_{21} & M_{22}
\end{array}\right],
\end{aligned}
$$

where

$$
\boldsymbol{M}_{\mathbf{1 1}}=\left[\begin{array}{ccc}
m_{1} & 0 & 0 \\
m_{2} & m_{2} & 0 \\
m_{3} & m_{3} & m_{3}
\end{array}\right],
$$

and

$$
M_{12}=M_{21}=M_{22}=0_{3,3}
$$

Further,

$$
K=\left[\begin{array}{ll}
K_{11} & K_{12} \\
K_{21} & K_{22}
\end{array}\right],
$$

where

$$
\begin{gathered}
\boldsymbol{K}_{11}=\left[\begin{array}{ccc}
\mathrm{a} k_{1} & -\mathrm{a} k_{2} & 0 \\
0 & \mathrm{a} k_{2} & -\mathrm{a} k_{3} \\
0 & 0 & \mathrm{a} k_{3}
\end{array}\right], \\
\boldsymbol{K}_{12}=\left[\begin{array}{ccc}
(1-\mathrm{a}) k_{1} & -(1-\mathrm{a}) k_{2} & 0 \\
0 & (1-\mathrm{a}) k_{2} & -(1-\mathrm{a}) k_{3} \\
0 & 0 & (1-\mathrm{a}) k_{3}
\end{array}\right],
\end{gathered}
$$

and

$$
\boldsymbol{K}_{21}=\boldsymbol{K}_{22}=0_{3,3}
$$

In Eqs. (54) and (55) stands for the rigidity ratio which can be viewed as a form of post-yield to preyield stiffness ratio $(a=1$ corresponds to the linear system). Further, the damping matrix of the structural system $C$ is assumed to be proportional to the stiffness matrix; that is,

$$
C=\left[\begin{array}{ll}
C_{11} & C_{12} \\
C_{21} & C_{22}
\end{array}\right],
$$

where

$$
\begin{gathered}
C_{11}=c K_{11}, \\
C_{12}=C_{21}=0_{3,3},
\end{gathered}
$$

and

$$
\boldsymbol{C}_{22}=\left[\begin{array}{lll}
1 & 0 & 0 \\
0 & 1 & 0 \\
0 & 0 & 1
\end{array}\right] .
$$

In Eq. (58) $c$ is taken equal to $0.2 \times 10^{-2}$. For the specific example $\gamma_{i}=m_{i}$, and the loading vector becomes

$$
\boldsymbol{F}(t)^{T}=\left(f_{1}(t) f_{2}(t) f_{3}(t) 0000\right) .
$$

Further,

$$
\begin{aligned}
& \boldsymbol{g}(\boldsymbol{y}, \dot{\boldsymbol{y}})^{\boldsymbol{T}}= \\
& =\left(\begin{array}{lll}
0 & 0 & 0
\end{array}-g_{1}\left(\dot{y}_{1}, z_{1}\right)-g_{2}\left(\dot{y}_{2}, z_{2}\right)-g_{3}\left(\dot{y}_{3}, z_{3}\right)\right) .
\end{aligned}
$$

In the Bouc-Wen model the additional state $z_{i}$ is associated with the displacement $y_{i}$ via the equation:

$$
\dot{z}_{i}=g_{i}\left(\dot{y}_{i}, z_{i}\right) \text {, }
$$

where 


$$
g_{i}\left(\dot{y}_{i}, z_{i}\right)=-\gamma\left|\dot{y}_{i}\right| z_{i}\left|z_{i}\right|^{n-1}-\beta \dot{y}_{i}\left|z_{i}\right|^{n}+A \dot{y}_{i} .
$$

The parameters $\gamma, \beta, A$ and $n$ are capable of representing a wide range of hysteresis loops (e.g., [28] and [29]). In this example the values $\mathrm{a}=0.15$, $\beta=\gamma=0.5, n=1$ and $A=1$ are considered. The equivalent linear matrices take the form (e.g., [1] to [3]):

$$
C_{e q}=\left[\begin{array}{ll}
C_{e q 11} & C_{e q 12} \\
C_{e q 21} & C_{e q 22}
\end{array}\right],
$$

where

$$
C_{e q 11}=C_{e q 12}=C_{e q 22}=0_{3,3},
$$

and

$$
\boldsymbol{C}_{e q 21}=\left[\begin{array}{ccc}
c_{e q 1} & 0 & 0 \\
0 & c_{e q 2} & 0 \\
0 & 0 & c_{e q 3}
\end{array}\right]
$$

Further,

$$
K_{e q}=\left[\begin{array}{ll}
K_{e q 11} & K_{e q 12} \\
K_{e q 21} & K_{e q 22}
\end{array}\right],
$$

where

$$
K_{e q 11}=K_{e q 12}=K_{e q 21}=0_{3,3}
$$

and

$$
\boldsymbol{K}_{e q 22}=\left[\begin{array}{ccc}
k_{e q 1} & 0 & 0 \\
0 & k_{e q 2} & 0 \\
0 & 0 & k_{e q 3}
\end{array}\right] .
$$

The elements $c_{e q_{i}}$ and $k_{e q_{i}}$ in Eqs. (67) and (70) are given by the expressions:

$$
c_{e q_{i}}=\sqrt{\frac{2}{\pi}}\left[\gamma \frac{E\left(\dot{y}_{i} z_{i}\right)}{\sqrt{E\left(\dot{y}_{i}^{2}\right)}}+\beta \sqrt{E\left(z_{i}^{2}\right)}\right]-A,
$$

and

$$
k_{e q_{i}}=\sqrt{\frac{2}{\pi}}\left[\gamma \sqrt{E\left(\dot{y}_{i}^{2}\right)}+\beta \frac{E\left(\dot{y}_{i} z_{i}\right)}{\sqrt{E\left(z_{i}^{2}\right)}}\right],
$$

respectively.

\subsection{A 3-DOF Hysteretic System under Evolutionary Stochastic Excitation of the Separable Form}

In this example, the excitation EPS $S_{\ddot{\alpha}}(\omega, t)$ takes the form

$$
S_{\ddot{\alpha}}(\omega, t)=|w(t)|^{2} S_{C P}(\omega),
$$

where $S_{C P}(\omega)$ represents the widely used in engineering applications Clough-Penzien power spectrum (e.g., [31]) and $w(t)$ denotes a timemodulating envelope function given by:

$$
w(t)=k\left(e^{-b_{1} t}-e^{-b_{2} t}\right),
$$

where $b_{1}=0.1$ and $b_{2}=0.3$; and $k$ is a normalization constant so that $w(t)_{\max }=1$. The Clough-Penzien spectrum is given by:

$$
\begin{aligned}
S_{C P}(\omega) & =S_{0} \frac{\omega_{g}{ }^{4}+4\left(\xi_{g}\right)^{2} \omega_{g}{ }^{2} \omega^{2}}{\left(\omega_{g}{ }^{2}-\omega^{2}\right)^{2}+4 \xi_{g}{ }^{2} \omega_{g}{ }^{2} \omega^{2}} \times \\
& \times \frac{\left(\omega / \omega_{f}\right)^{4}}{\left(1-\left(\omega / \omega_{f}\right)^{2}\right)^{2}+4 \xi_{f}{ }^{2}\left(\omega / \omega_{f}\right)^{2}}
\end{aligned}
$$

where $S_{0}$ is the amplitude of the excitation spectrum, modeled as a white noise process. The parameters values used are $\xi_{g}=0.7, \omega_{g}=2 \mathrm{rads}^{-1}, \xi_{f}=0.6$, $\omega_{f}=12.5 \mathrm{rad} \mathrm{s}^{-1}$. The total duration of the excitation is 20 seconds. Further, the hysteretic 3-DOF system has the properties $m_{1}=2.0615 \times 10^{5} \mathrm{~kg}, m_{2}=2.0559 \times 10^{5} \mathrm{~kg}$, $m_{3}=2.0261 \times 10^{5} \mathrm{~kg}, \quad k_{1}=3.9668 \times 10^{8} \mathrm{Nm}^{-1}$, $k_{2}=3.5007 \times 10^{8} \mathrm{Nm}^{-1}$ and $k_{3}=2.6927 \times 10^{8} \mathrm{Nm}^{-1}$. In Fig. 1 the EPS of $S_{\ddot{\alpha}}(\omega, t)$ is plotted for $S_{0}=20 \mathrm{~m}^{2} \mathrm{~s}^{-3}$.

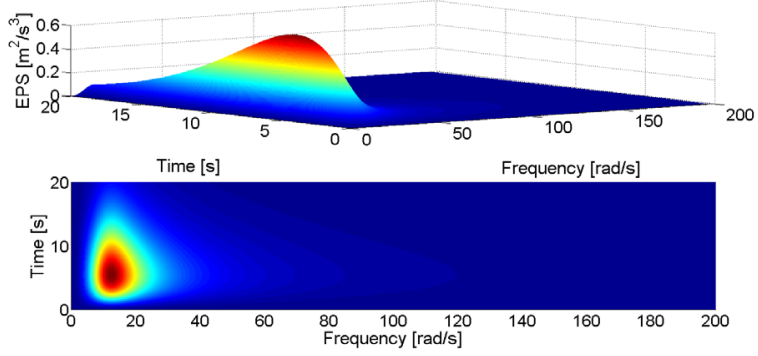

Fig. 1. Separable excitation evolutionary power spectrum

In Figs. 2 and 3 the equivalent time-varying natural frequency $\omega_{\text {eq,i }}(t)$ and $\beta_{\text {eq,i } i}(t)$ the damping element corresponding to each DOF are plotted, respectively. Note that the hysteretic/degrading behavior of the system is captured by the decreasing with time trend of the stiffness element, as well as the increasing with time trend of the damping element.

Further, in Figs. 4 and 5 the survival probabilities $P_{i}^{B}(T)$ and indicatively the corresponding firstpassage PDFs $p_{i}^{B}(T)$ for the first DOF of the hysteretic MDOF system are plotted for various barrier levels, respectively. The value $N=30$ is chosen regarding the number of terms to be included in Eq. (45). Comparisons between the analytical approximate technique and MCS data (10,000 realizations) 


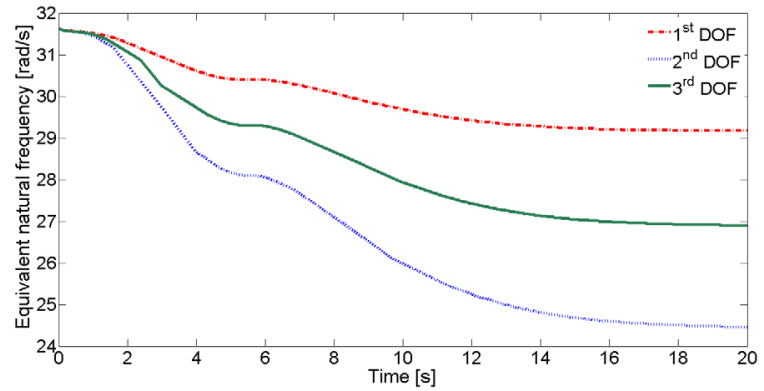

Fig. 2. Equivalent natural frequency $\omega_{\text {eq, } i}(t)$

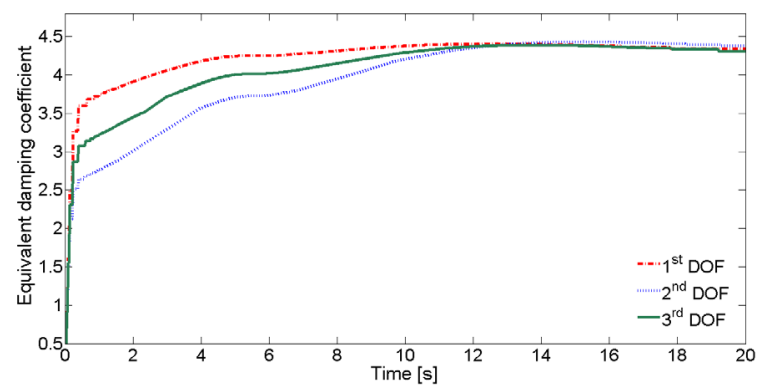

Fig. 3. Equivalent damping coefficient $\beta_{\text {eq, } i}(t)$

demonstrate a satisfactory degree of agreement. Note that the irregular/non-smooth shape of the approximate technique based first-passage PDFs is due to the differentiation of the survival probability (Eq. (37)). In this regard, the survival probability Eq. (40) is assumed to have constant values over the time intervals $\left[t_{i, j-1}, t_{i, j}\right]$, resulting in a non-smooth representation. Obviously, the level of nonsmoothness increases when differentiation takes place.

Furthermore, in Figs. 6 and 7 the survival probabilities $P_{i}^{B}(T)$ corresponding to the second and third DOF of the system are plotted for various barrier levels. Comparisons with MCS demonstrate a satisfactory degree of accuracy for these cases as well.

\subsection{A 3-DOF Hysteretic System under Evolutionary Stochastic Excitation of the Non-Separable Form}

The excitation EPS $S_{\ddot{\alpha}}(\omega, t)$ is assumed to have the non-separable form:

$$
S_{\ddot{\alpha}}(\omega, t)=S_{0}\left(\frac{\omega}{15 \pi}\right)^{2} e^{-b t} t^{2} e^{-\left(\frac{\omega}{15 \pi}\right)^{2} t},
$$

with $S_{0}=10 \mathrm{~m}^{2} \mathrm{~s}^{-3}$ and $b=0.5$. This spectrum comprises some characteristics of particular interest, such as decreasing of the dominant frequency with respect to

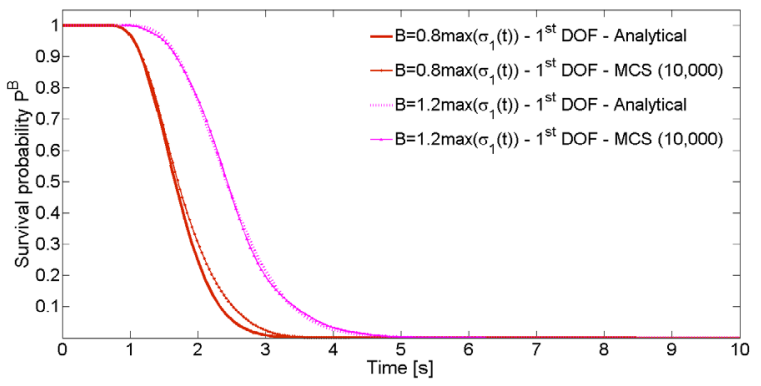

Fig. 4. Survival probability for various values of the parameter $\lambda$ for the first DOF; comparisons with MCS (10,000 realizations)

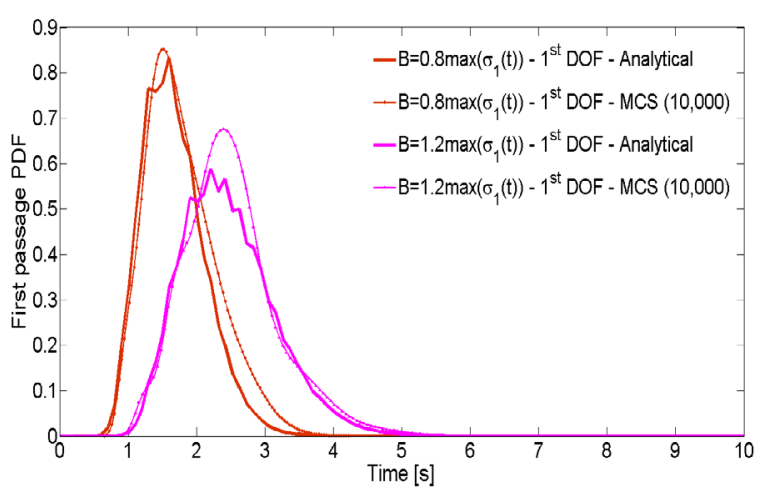

Fig. 5. First-passage PDF for various values of the parameter $\lambda$ for the first DOF; comparisons with MCS (10,000 realizations)

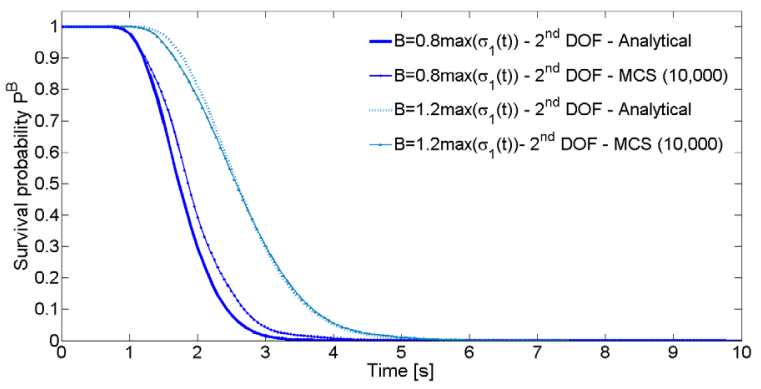

Fig. 6. Survival probability for various values of the parameter $\lambda$ for the second DOF; comparisons with MCS $(10,000$ realizations)

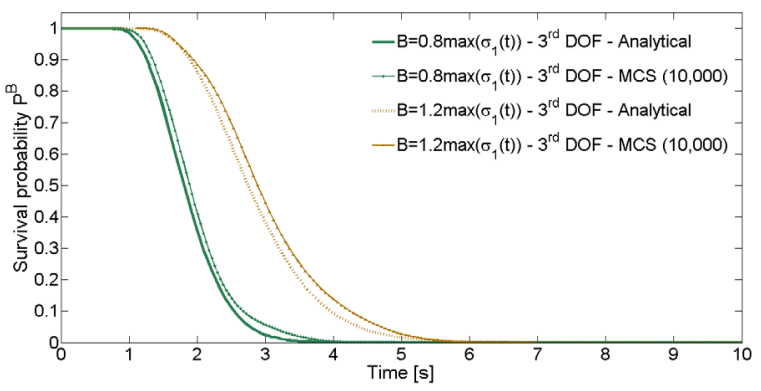

Fig. 7. Survival probability for various values of the parameter $\lambda$ for the third DOF; comparisons with MCS (10,000 realizations) 
time (e.g., [25] and [32]). Further, the hysteretic 3-DOF system parameters take the values $m_{1}=1.0240 \times 10^{5} \mathrm{~kg}$, $m_{2}=1.0225 \times 10^{5} \mathrm{~kg}, \quad m_{3}=1.0105 \times 10^{5} \mathrm{~kg} \quad$ and $k_{1}=5.6889 \times 10^{7} \mathrm{Nm}^{-1}, \quad k_{2}=5.6889 \times 10^{7} \mathrm{Nm}^{-1} \quad$ and $k_{3}=4.3945 \times 10^{7} \mathrm{Nm}^{-1}$. In Fig. 8 the non-separable excitation EPS is plotted.
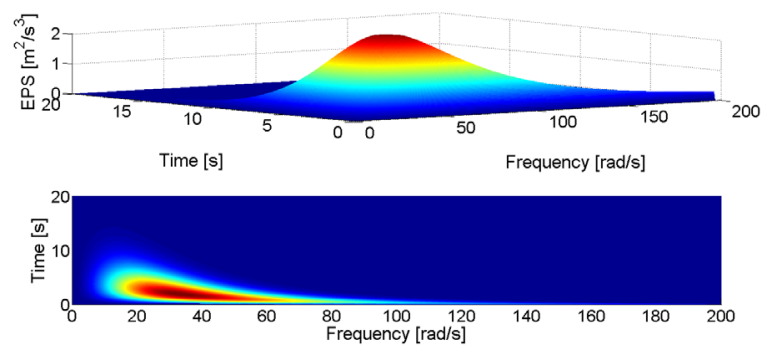

Fig. 8. Non-separable excitation evolutionary power spectrum $S_{\ddot{\alpha}}(\omega, t)$

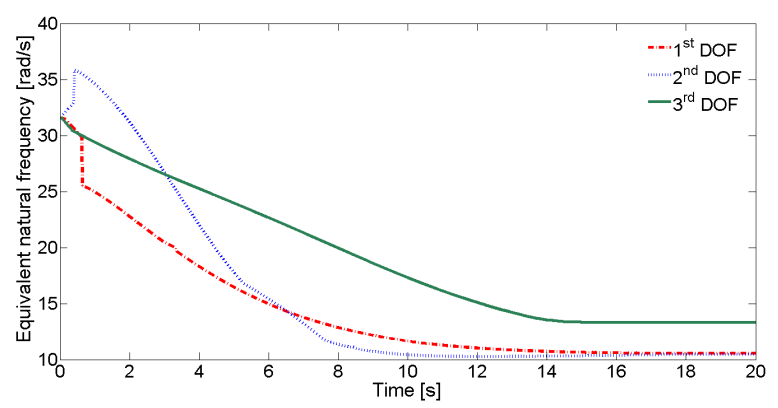

Fig. 9. Equivalent natural frequency $\omega_{e q, i}(t)$

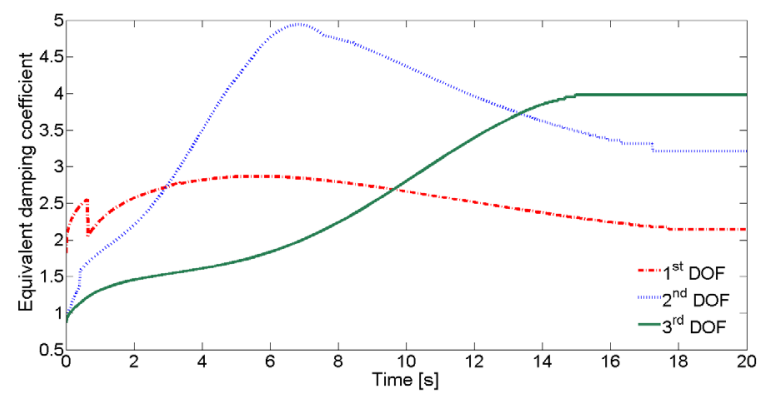

Fig. 10. Equivalent damping coefficient $\beta_{\text {eq,i }}(t)$

In Figs. 9 and 10 the equivalent time-varying natural frequency $\omega_{e q, i}(t)$ and damping $\beta_{e q, i}(t)$ elements corresponding to each DOF are plotted, respectively. Underlying the analytical approximate approach is the attempt to capture the time evolution as well as the essential characteristics of the frequency content of the nonlinear system response. Note that the ability of the technique to provide with time-varying natural frequencies $\omega_{e q, i}(t)$ can be of particular importance if seen in conjunction with recent theoretical developments regarding the concept of the mean instantaneous frequency (MIF) (e.g., [33] to [35]). In this regard, $\omega_{e q, i}(t)$ together with the MIF of the excitation can be potentially employed for evaluating the effects of temporal non-stationarity in the frequency content of the excitation on the system response as well as for tracking moving resonance phenomena (e.g., [23] and [36]).

Further, in Figs. 11, 12 and 13 the survival probabilities $P_{i}^{B}(T)$ for every DOF of the hysteretic MDOF system are plotted for various barrier levels, respectively; comparisons with MCS $(10,000$ realizations) demonstrate a satisfactory degree of accuracy.

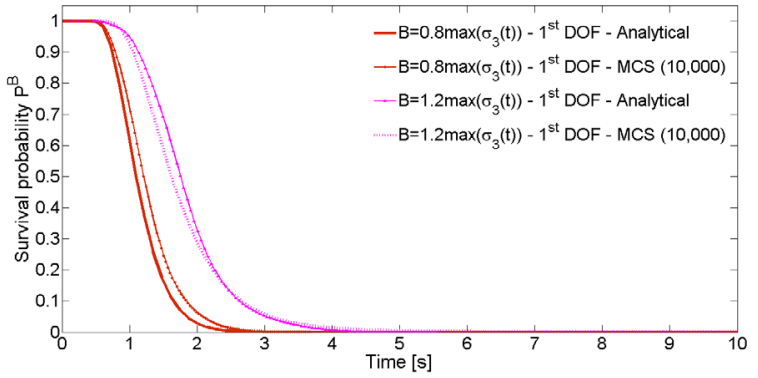

Fig. 11. Survival probability for various values of the parameter $\lambda$ for the first DOF; comparisons with MCS (10,000 realizations)

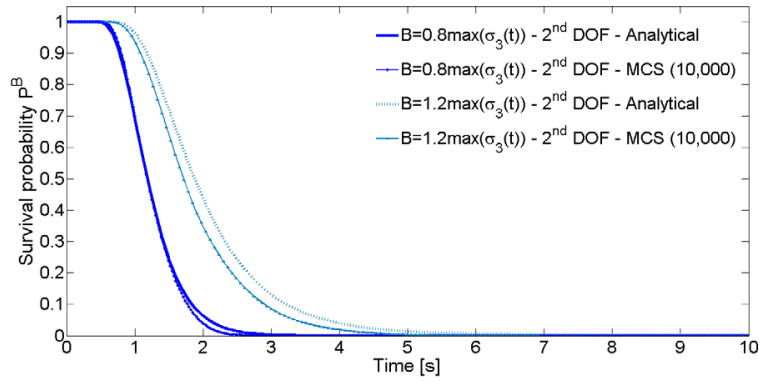

Fig. 12. Survival probability for various values of the parameter $\lambda$ for the second DOF; comparisons with MCS (10,000 realizations)

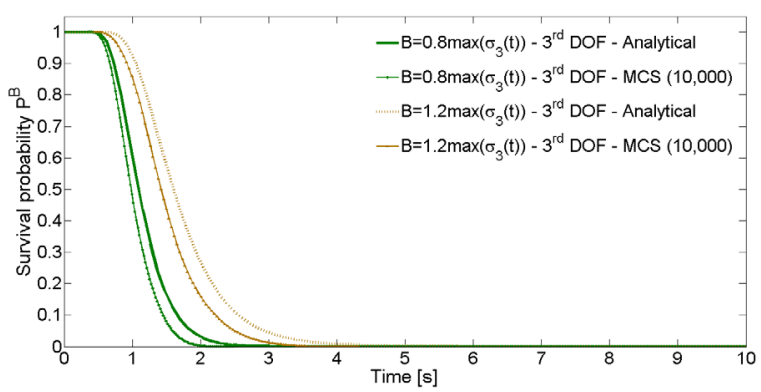

Fig. 13. Survival probability for various values of the parameter $\lambda$ for the third DOF; comparisons with MCS (10,000 realizations) 


\section{CONCLUDING REMARKS}

An approximate analytical technique for determining the time-varying survival probability and associated first-passage PDF of nonlinear/hysteretic MDOF systems subject to evolutionary stochastic excitation has been developed. Specifically, based on an efficient dimension reduction approach and relying on the concepts of stochastic averaging and statistical linearization, the original nonlinear $n$-degree-offreedom system has been decoupled and cast into $(n)$ effective single-degree-of-freedom (SDOF) linear time-variant (LTV) oscillators corresponding to each and every DOF. In this regard, time-varying effective stiffness $\omega_{e q, i}^{2}(t)$ and damping $\beta_{e q, i}(t)$ elements corresponding to each and every DOF have been defined and computed, while the non-stationary marginal, transition and joint response amplitude PDFs have been efficiently determined in closed-form expressions. Finally, the MDOF system survival probability and first-passage PDF have been determined approximately in a computationally efficient manner. Overall, the developed technique exhibits enhanced versatility since it can handle readily a wide range of nonlinear behaviors as well as various stochastic excitations with arbitrary nonseparable EPS forms that exhibit strong variability in both the intensity and the frequency content. A 3-DOF system exhibiting hysteresis following the Bouc-Wen model has been included in the numerical examples section. Comparisons with pertinent Monte Carlo simulations have demonstrated the reliability of the technique.

\section{ACKNOWLEDGEMENTS}

The first author acknowledges the financial support of the State Scholarships Foundation - IKY in Greece.

\section{REFERENCES}

[1] Roberts J.B., Spanos P.D. (2003). Random Vibration and Statistical Linearization. Dover Publications, New York.

[2] Soong, T.T., Grigoriu, M. (1993). Random Vibrations of Mechanical and Structural Systems. Prentice-Hall, New Jersey.

[3] Li, J., Chen, J. (2009). Stochastic Dynamics of Structures, Dover Publications, New York, Dol:10.1002/9780470824269.

[4] Schueller, G.I., Pradlwarter, H.J., Koutsourelakis, P.S. (2004). A critical appraisal of reliability estimation procedures for high dimensions. Probabilistic Engineering Mechanics, vol. 19, no. 4, p. 463-474, D0l:10.1016/j.probengmech.2004.05.004.

[5] Corotis, R., Vanmarcke, E.H., Cornell, C.A. (1972). First passage of nonstationary random processes. Journal of the Engineering Mechanics Division, vol. 98, no. 2, p. 401-414.
[6] Vanmarcke, E.H. (1975). On the distribution of the firstpassage time for normal stationary random processes. ASME Journal of Applied Mechanics, vol. 42, no. 1, p. 215-220, DOI:10.1115/1.3423521.

[7] Barbato, M., Conte, J.P. (2001). Structural reliability applications of non-stationary spectral characteristics. ASCE Journal of Engineering Mechanics, vol. 137, no. 5, p. 371-382, DOI:10.1061/(ASCE)EM.1943-7889.0000238.

[8] Kovaleva, A. (2009). An exact solution of the first-exit time problem for a class of structural systems. Probabilistic Engineering Mechanics, vol. 24, no. 3, p. 463-466, D0I:10.1016/j.probengmech.2009.01.002.

[9] Solomos, G.P., Spanos, P.D. (1983). Structural reliability under evolutionary seismic excitation. International Journal of Soil Dynamics and Earthquake Engineering, vol. 2, no. 2, p. 110116, D0I:10.1016/0261-7277(83)90007-4.

[10] lourtchenko, D.V., Mo, E., Naess, A. (2006). Response probability density functions of strongly non-linear systems by the path integration method. International Journal of NonLinear Mechanics, vol. 41, no. 5, p. 693-705, D0l:10.1016/j. ijnonlinmec.2006.04.002.

[11] Naess, A., lourtchenko, D., Batsevych, O. (2011). Reliability of systems with randomly varying parameters by the path integration method. Probabilistic Engineering Mechanics, vol. 26, no. 1, p. 5-9, D0l:10.1016/j.probengmech.2010.05.005.

[12] Kougioumtzoglou, I.A., Spanos, P.D. (2013). Response and first-passage statistics of nonlinear oscillators via a numerical path integral approach. ASCE Journal of Engineering Mechanics, vol. 139, no. 9, p. 1207-1217, Dol:10.1061/ (ASCE)EM.1943-7889.0000564.

[13] Kougioumtzoglou, I.A., Spanos, P.D. (2014). Stochastic response analysis of the softening Duffing oscillator and ship capsizing probability determination via a numerical path integral approach. Probabilistic Engineering Mechanics, vol. 35, p. 67-74, D0I:10.1016/j.probengmech.2013.06.001.

[14] Spanos, P.D, Kougioumtzoglou, I.A. (2014). Survival probability determination of nonlinear oscillators subject to evolutionary stochastic excitation. Journal of Applied Mechanics, vol. 81, no. 5, 051016, D0l:10.1115/1.4026182.

[15] Spanos, P.D., Lutes, L.D. (1980). Probability of response to evolutionary process. Journal of Engineering Mechanics, vol. 106 , no. 2, p. 213-224.

[16] Kougioumtzoglou, I.A., Spanos, P.D. (2013). Nonlinear MDOF system stochastic response determination via a dimension reduction approach. Computers and Structures, vol. 126, p. 135-148, D0l:10.1016/j.compstruc.2012.11.020.

[17] Priestley, M.B. (1965). Evolutionary spectra and non-stationary processes. Journal of the Royal Statistical Society, vol. 27, no. 2, p. 204-237.

[18] Dahlhaus, R. (1997). Fitting time series models to nonstationary processes. The Annals of Statistics, vol. 25, no. 1, p. 1-37, DOl:10.1214/aos/1034276620.

[19] Gajic, Z., Qureshi, M. (1995). Lyapunov Matrix Equation in System Stability and Control. Academic Press, New York.

[20] Mitseas, I.P., Kougioumtzoglou, I.A., Beer, M. (2016). An approximate stochastic dynamics approach for nonlinear structural system performance-based multi-objective optimum 
design. Structural Safety, vol. 60, p. 67-76, D0l:10.1016/j. strusafe.2016.01.003.

[21] Hammond, J.K. (1973). Evolutionary spectra in random vibration. Journal of the Royal Statistical Society, vol. 35, no. 2, p. 167-188.

[22] Jangid, R.S., Datta, T.K. (1999). Evaluation of the methods for response analysis under non-stationary excitation. Shock and Vibration, vol. 6, no. 5-6, p. 285-297, DOI:10.1155/1999/312010.

[23] Tubaldi, E., Kougioumtzoglou, I.A. (2015). Nonstationary stochastic response of structural systems equipped with nonlinear viscous dampers under seismic excitation. Earthquake Engineering \& Structural Dynamics, vol. 44, no. 1, p. 121-138, Dol:10.1002/eqe.2462.

[24] Giaralis, A., Spanos, P.D. (2010). Effective linear damping and stiffness coefficients of nonlinear systems for design spectrum based analysis. Soil Dynamics and Earthquake Engineering, vol. 30, no. 9, p. 798-810, Dol:10.1016/j.soildyn.2010.01.012.

[25] Spanos, P.D., Solomos, G.P. (1983). Markov approximation to transient vibration. Journal of Engineering Mechanics, vol. 109, no. 4, p. 1134-1150, Dol:10.1061/(ASCE)07339399(1983)109:4(1134).

[26] Spanos, P.D. (1978). Non-stationary random vibration of a linear structure. International Journal of Solids and Structures, vol 14, no. 10, p. 861-867, D0l:10.1016/00207683(78)90076-8.

[27] Abramowitz, M., Stegun, I.A. (1970). Handbook of Mathematical Functions. Dover Publications, New York.

[28] Wen, Y.K. (1980). Equivalent linearization for hysteretic systems under random excitation. Journal of Applied Mechanics, vol. 47, no. 1, p. 150-154, Dol:10.1115/1.3153594.
[29] Ikhouane, F., Rodellar, J. (2007). Systems with Hysteresis: Analysis, Identification and Control using the Bouc-Wen Model. John Wiley and Sons, Chichester, DOl:10.1002/9780470513200.

[30] Shinozuka, M., Deodatis, G. (1991). Simulation of stochastic processes by spectral representation. Applied Mechanics Reviews, vol. 44, no. 4, p. 191-204, D0l:10.1115/1.3119501.

[31] Clough, R.W., Penzien, J. (1993). Dynamics of Structures, McGraw-Hill, New York.

[32] Liu, S.C. (1970). Evolutionary power spectral density of strongmotion earthquakes. Bulletin of the Seismological Society of America, vol. 60, no. 3, p. 891-900.

[33] Qian, S. (2002). Introduction to Time-Frequency and Wavelet Transforms. Prentice Hall, New Jersey.

[34] Kijewski-Correa, T., Kareem, A. (2006). Efficacy of Hilbert and wavelet transforms for time-frequency analysis. Journal of Engineering Mechanics, vol. 132, no. 10, p. 1037-1049, DOl:10.1061/(ASCE)0733-9399(2006)132:10(1037).

[35] Spanos, P.D., Giaralis, A., Politis, N.P., Roesset, J.M. (2007). Numerical treatment of seismic accelerograms and of inelasticseismic structural responses using harmonic wavelets. Computer-Aided Civil and Infrastructure Engineering, vol. 22, no. 4, p. 254-264, Dol:10.1111/j.14678667.2007.00483.x.

[36] Beck, J.L., Papadimitriou, C. (1993). Moving resonance in nonlinear response to fully non-stationary stochastic ground motion. Probabilistic Engineering Mechanics, vol. 8, no. 3-4, p. 157-167, DOl:10.1016/0266-8920(93)90011-J. 